RESEÑA

\title{
SILVICULTURA DE PLANTACIONES FORESTALES COMERCIALES
}

Título: Silvicultura de plantaciones forestales comerciales.

Autor: Miguel Ángel Musálem.

Edición: Universidad Autónoma Chapingo, División de Ciencias Forestales, Departamento de Ecología y Silvicultura.

No. de páginas: 174 .

Año: 2002.

En las primeras etapas de la civilización humana, la necesidad de madera de los escasos pobladores estaba cubierta, por lo que, los recursos naturales permanecían casi inalterados. Conforme la población humana creció, aumentó también la necesidad de madera tanto para combustible como para construcción; sin embargo, los bosques naturales siguieron proporcionando, por mucho tiempo, la madera necesaria para tales fines.

Para suplir las necesidades crecientes de madera para uso industrial relacionado con el crecimiento de la población, surgió el concepto del aprovechamiento sostenido de los recursos forestales. No obstante, los grandes esfuerzos por obtener de los bosques naturales la madera necesaria para satisfacer los requerimientos mínimos de la población en forma sostenida, éstos no fueron suficientes. De aquí, surge una gran presión sobre los bosques naturales, que propició la destrucción de grandes áreas forestales. Por otro lado, el cambio de uso de grandes áreas forestales convertidas a terrenos agrícolas y ganaderos redujo dramáticamente las superficies forestales, que trajo como consecuencia el deterioro del ambiente. El complejo tejido; social, económico y político que genera el crecimiento poblacional, ha creado sobre el recurso forestal una gran presión, que ha generado destrucción, deterioro de la calidad del ambiente y baja productividad de los recursos forestales. Ante esta situación, surge la necesidad de plantar árboles no sólo con el objeto de satisfacer las 
necesidades de madera, sino también como una forma de mejorar el ambiente y la calidad de la vida.

Sin embargo, la demanda creciente de bienes y servicios de la sociedad, así como los de conservación de su ambiente y recursos asociados, ha llevado a la creación de bosques llamados artificiales para sustituir de manera más cercana sus necesidades, sobre todo de productos industriales. A este punto, aunque las plantaciones originalmente fueron encaminadas para sustituir los casos de falla de la regeneración de los bosques naturales y para restaurar algunas áreas afectadas por daños ocasionados por el hombre o accidentes naturales; han crecido como una alternativa de producción intensiva más controlada en cuanto a la uniformidad de sus productos y la mayor productividad por unidad de superficie.

Debido a la importancia y a la escasez de información sobre la silvicultura de plantaciones forestales comerciales, el autor aborda un total de 10 capítulos que fueron inteligentemente pensados siguiendo una secuencia lógica, de tal manera que el lector va adquiriendo el conocimiento necesario en forma paulatina.

El libro arranca con el capítulo sobre la "necesidad de las plantaciones forestales" en el que se describen algunos de los factores más importantes acerca de la necesidad de plantar árboles, entre los más relevantes se mencionan los siguientes: la destrucción del recurso forestal; el crecimiento de la demanda de madera; los problemas de la regeneración natural; la lejanía y poca accesibilidad de los bosques naturales a centros de consumo, y el deterioro ambiental. Además que permite al lector ubicarse en el campo de acción de las plantaciones forestales, conocer la situación de la actividad en el mundo y en México, reconociendo las tendencias de la creación de los bosques artificiales en relación a la utilización y conservación de los bosques naturales, su papel en el desarrollo industrial, así como distinguir los distintos tipos de plantaciones forestales de acuerdo a sus objetivos.

En el capítulo dos se aborda el tema de "elección de especies" a utilizar en una plantación forestal, en primer lugar, es necesario detallar los objetivos que persigue 
una plantación. Sigue con un análisis de cómo afectan las características del sitio (clima, suelo, factores bióticos, entre otros) en la elección de las especies, para posteriormente, señalar el manejo silvícola a realizar. Dependiendo del tipo de plantación, será necesario ponderar algunos aspectos, por ejemplo: en las plantaciones comerciales, lo más importante es la especie, la cual tiene que cumplir con el objetivo de la plantación; en las plantaciones escénicas, lo que importa es el objetivo $\mathrm{y}$, en este caso, existen una multitud de especies para dichos fines; en las demás plantaciones, lo que importa es la especie, de ahí que es necesario tomar en cuenta los objetivos y los sitios donde tenga que realizarse la plantación. Además de realizar una discusión sobre las ventajas que ofrecen las especies exóticas y nativas de acuerdo a las condiciones de cada lugar de plantación.

En el capítulo tres se discuten los "métodos de plantación" más importantes para establecer las plantaciones forestales de acuerdo al tipo de terreno y las condiciones de desarrollo económico y social. Así mismo, en este capítulo, se describe la necesidad de un levantamiento previo a la plantación que sirva como guía para la toma de decisiones en aspectos tales como infraestructura, condiciones edáficas y topográficas, luego, se analizan los tipos de vegetación y cómo influyen en los futuros trabajos de preparación del sitio. Se sigue con un análisis del tipo y condiciones de terreno con el fin de describir las formas de laboreo previas a la plantación para, posteriormente, continuar con la descripción de la plantación de brinzales.

En el capítulo cuatro se describen los "aclareos en plantaciones forestales industriales" estos tratamientos intermedios que se aplican a las plantaciones a lo largo del turno de producción. Se enfatiza la aplicación de los aclareos para manejo óptimo de plantación y para producción de productos intermedios. Se hace especial énfasis en los métodos de aclareos para plantaciones forestales industriales.

En el capítulo cinco se enfatizan sobre el "cultivo de las plantaciones" tema fundamental en el manejo del crecimiento y protección de la plantación desde que se establece hasta que llega el tiempo de ser aprovechada, tales como las limpiezas, las 
podas y la protección a los agentes externos de daño. Una vez establecida la plantación, los cuidados posteriores para su adecuada protección y crecimiento. Esta fase de cultivo, desde la plantación hasta la cosecha, cuando se ha soslayado, ha resultado en fracasos que desalientan toda la operación de plantación. El programa de cultivo incluye normalmente la sustitución de plantas muertas durante el primer período anual de crecimiento conocida como reposición de fallas; el control de la vegetación competidora o control de malezas; la fertilización; y desde luego la protección.

En el capítulo seis sobre el "impacto ecológico de las plantaciones forestales comerciales", se hace una revisión de los impactos benéficos de las plantaciones desde el punto de vista del mejoramiento del ambiente y la conservación de otros recursos, así como también, se enfatiza la revisión de las posibilidades de impactos negativos sobre la conservación de la biodiversidad. Una revisión exhaustiva de los impactos ecológicos de las plantaciones forestales comerciales está fuera del alcance de este capítulo, en el que se discuten de manera general algunos de los mitos y realidades sobre este tema, con base en información disponible.

En el capítulo siete se presenta un resumen de los "aspectos económicos y financieros de las plantaciones forestales comerciales" que generan la producción de celulosa en México, así como su relación con los bosques naturales y las plantaciones forestales comerciales. Es importante hacer mención que nuestro país es deficitario en su producción de celulosa, que es la materia prima principal para la producción de papel y cartón, lo que hace especialmente importante revisar estos temas para la planeación de establecimiento de plantaciones forestales comerciales.

En el capítulo ocho se hace una revisión sobre la "evaluación financiera de plantaciones forestales comerciales" de sus indicadores económicos y financieros, se realiza su cálculo y se presentan programas existentes para obtenerlos, como una base esencial para la toma de decisiones sobre proyectos de éste tipo. La evaluación puede realizarse, al menos, bajo dos enfoques: el económico y el financiero, para determinar si una propuesta es apropiada y tiene bases firmes, antes de comprometer 
los recursos a invertir. Así, la evaluación de plantaciones se inicia cuando es indispensable conocer el valor de algunas variables para una acertada toma de decisiones. En México, la evaluación de plantaciones es reciente, la mayor parte de éstas se ha realizado desde los años sesenta y, muy esporádicamente, en años anteriores. La mayoría de las evaluaciones realizadas consideran solamente variables dasométricas y, sólo hasta fechas recientes, se han evaluado financieramente; teniendo un mayor auge las evaluaciones de plantaciones experimentales.

Con las reformas al Artículo 27 Constitucional, la nueva Ley Agraria y la nueva Ley Forestal se abren las expectativas de fomento a las plantaciones forestales. Estos cambios conciben la formación de sociedades comerciales, las cuales podrán ser propietarias de terrenos rústicos en la extensión que sea necesaria para el cumplimiento de su objetivo, así como también dota de plena personalidad jurídica a los núcleos de población ejidales, con lo cual se establece los procedimientos por los cuales los ejidatarios y comuneros podrán asociarse entre sí, con el estado y con terceros, y otorgar el uso de las tierras. En relación a lo mencionado anteriormente, en los dos últimos capítulos del libro se presentan los programas de "incentivos para el establecimiento de las plantaciones forestales comerciales", así como "la normatividad de las plantaciones" que regula su establecimiento y aprovechamiento de acuerdo con la nueva ley de Desarrollo Forestal Sustentable y su reglamento.

Como conclusión final, podemos afirmar que esta obra supone un referente para todos aquellos que se interesan por el campo de la silvicultura de plantaciones forestales comerciales, no sólo para los investigadores, sino también para los prestadores de servicios técnicos forestales y productores forestales del país.

Rosa Martínez Ruiz. Doctora en Ciencias Forestales. Profesor investigador. Ingeniería en Sistemas Forestales. Universidad Autónoma Indígena de México. Correo electrónico: ruizrosa@uaim.edu.mx. 